\title{
Lower hybrid current drive experiments in support of high confinement long pulse operation in EAST
}

\author{
Annika Ekedahl ${ }^{1, *}$, Bojiang Ding ${ }^{2}$, Xianzu Gong ${ }^{2}$, Marc Goniche ${ }^{1}$, Miaohui Li $^{2}$, Yves Peysson ${ }^{1}$, Jinping Qian ${ }^{2}$, \\ Julien Hillairet ${ }^{1}$, Tuong Hoang ${ }^{1}$, Fukun Liu ${ }^{2}$, Chengming Qin $^{2}$, Yuantao Song ${ }^{2}$, Xiaojie Wang ${ }^{2}$, Xinjun Zhang $^{2}$, \\ Yanping Zhao ${ }^{2}$, Xiao-Lan Zou ${ }^{1}$ and the EAST Team ${ }^{2}$ \\ ${ }^{1}$ CEA, IRFM, F-13108 Saint Paul-lez-Durance, France. \\ ${ }^{2}$ Institute of Plasma Physics, Chinese Academy of Sciences, Hefei 230031, P. R. China.
}

\begin{abstract}
The lower hybrid current drive (LHCD) system plays a crucial role in the mission of the Experimental Advanced Superconducting Tokamak (EAST) and is a prerequisite for reaching long pulse, high confinement plasmas on EAST [1, 2]. LHCD experiments and modelling [3] have been carried out on EAST in 2015-2016, with the aim to optimising EAST long pulse scenarios, and at the same time gain experience for the exploitation of WEST [4]. Experiments have been carried out to study the LH current drive efficiency in different plasma configurations (Upper Single Null and Lower Single Null). The effect of the gas feed location on the LH wave coupling was investigated by comparing gas fuelling from high field side, low field side and upper divertor. In view of long pulse $\mathrm{H}$-mode scenarios, a series of H-mode experiments were conducted where all the heating power was provided by RF heating methods only, i.e. LHCD, ECRH and ICRH. H-modes were sustained in both Upper Single Null (W divertor) and Lower Single Null (carbon divertor) configurations, with loop voltage maintained as low as $50 \mathrm{mV}$.
\end{abstract}

\section{Introduction}

The radiofrequency (RF) heating and current drive systems play a crucial role in the mission of the Experimental Advanced Superconducting Tokamak (EAST) and are prerequisites for reaching long pulse, high-confinement (H-mode) plasmas on EAST [1, 2]. This paper reports on LHCD experiments and modelling carried out on EAST in 2015-2016, within the framework of the Associated Laboratory ASIPP-IRFM, with the aim to optimize EAST long pulse scenarios, and at the same time gain experience before the exploitation of the WEST device [4]. The unique Lower Hybrid Current Drive (LHCD) capability, i.e. two LHCD systems at different frequencies $(2.45 \mathrm{GHz}$ [5] and 4.6 $\mathrm{GHz}[6])$, has allowed comparing the CD efficiency of the two LHCD systems [7]. Extensive modelling with the ray-tracing + Fokker-Planck codes C3PO/LUKE [8] has been performed in support to the experiments [3]. Several experiments, mainly focusing on the $4.6 \mathrm{GHz}$ launcher, have been carried out to study the CD efficiency in different plasma configurations and with different gas feeding locations. In view of long pulse $\mathrm{H}$ mode scenarios, a series of $\mathrm{H}$-mode experiments has been conducted where all the heating power was provided by RF heating methods only, i.e. LHCD, ECRH (Electron Cyclotron Resonance Heating) and ICRH (Ion Cyclotron Resonance Heating).

In addition to the experimental results presented here, EAST experiments have revealed some operational

\footnotetext{
* Corresponding author: annika.ekedahl@cea.fr
}

issues important for long pulse operation. During the 2015 campaign, arcing at the $4.6 \mathrm{GHz}$ launcher, accompanied by emission of flakes near the launcher and divertor regions, was observed via visible cameras [9]. Following the in-vessel inspections, several corrective actions were taken before the 2016 campaign: i) new LH launcher guard limiters were installed; ii) the LH launcher mouth was positioned radially closer to the guard limiters in order to reduce the electric field at launcher mouth; iii) all in-vessel components were cleaned from deposits that had accumulated during the previous plasma operation campaign.

\section{LH wave coupling}

EAST uses two LHCD systems operating at different frequencies. The $2.45 \mathrm{GHz}$ launcher has 20 modules of eight waveguides, mounted in five rows and four columns [5]. The $4.6 \mathrm{GHz}$ launcher is composed of 24 modules of $3 \times 24$ waveguides, mounted in four rows and six columns [6]. The launched wave spectra for different phasing between modules for the $4.6 \mathrm{GHz}$ launcher are shown in Figure 1. The main peak is located at around $\mathrm{n}_{\mathrm{z}}=-2$ (negative $\mathrm{n}_{\mathrm{z}}$ corresponds to the co-current direction). Approximately $20 \%$ of the power is launched in the counter-current direction, but with a higher $n_{z}$ thus creating less energetic electrons in the counter-current direction. The launched wave spectra for the $2.45 \mathrm{GHz}$ launcher have quite similar properties as the $4.6 \mathrm{GHz}$ launcher, but with a larger width of the main peak. 


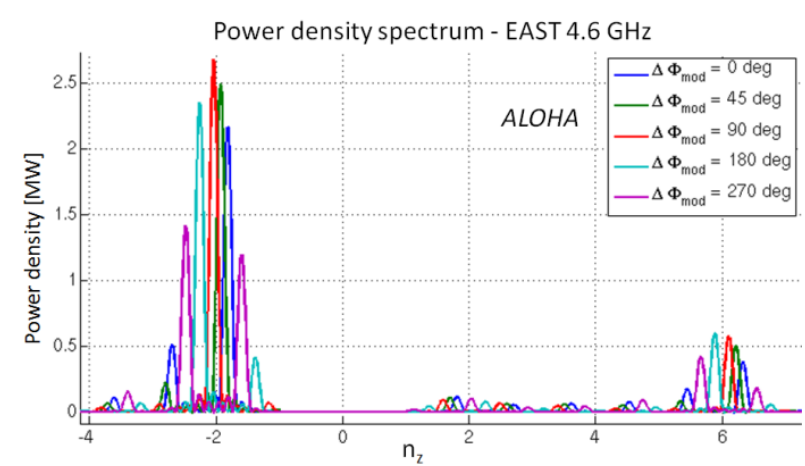

Fig. 1. Launched wave spectra for the EAST 4.6 GHz launcher, as computed with the ALOHA code [10].

The effect of particle fueling on the average power reflection coefficient (RC) of the $4.6 \mathrm{GHz}$ launcher was studied using gas injection from different locations, combined with Supersonic Molecular Beam Injection (SMBI). These experiments were carried out in the Upper Single Null (USN) configuration $\left(\mathrm{B}_{\mathrm{T}}=2.5 \mathrm{~T}, \mathrm{I}_{\mathrm{P}}=\right.$ $0.5 \mathrm{MA}$ ). Most of the particles were provided by the standard gas injection and the SMBI was used for the feedback control of the line averaged density which was ramped from $2.5 \times 10^{19} \mathrm{~m}^{-3}$ to $4 \times 10^{19} \mathrm{~m}^{-3}$. Three different gas injections were tested: high field side (HFS), low field side (LFS) and upper divertor). The RC is almost identical in the whole density range (Figure 2). It is noticeable that the $\mathrm{RC}$ is almost constant at $6 \%$ for quite a large part of the density ramp and then drops to $4 \%$ when the density exceeds $3.5 \times 10^{19} \mathrm{~m}^{-3}$. This drop is correlated with the phase of the discharge where the largest amount of particle fueling comes from SMBI, a method that has been found to increase radial transport [11]. This effect results in an increase in density in the far scrape-off layer, which is clearly beneficial for LH coupling.

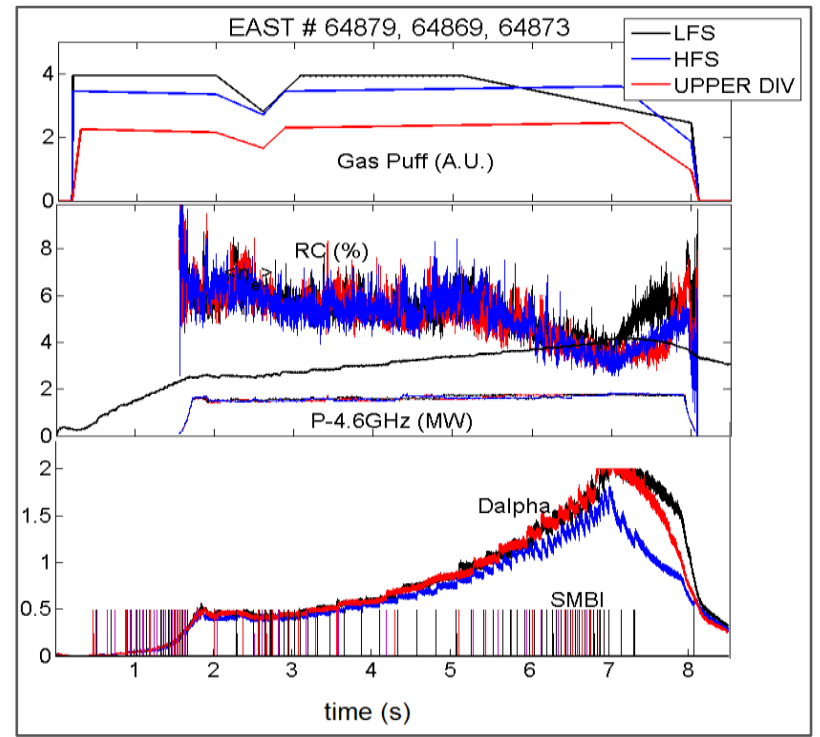

Fig. 2. Average reflection coefficient (RC) on the $4.6 \mathrm{GHz}$ launcher for different gas fueling locations.

\section{LHCD efficiency}

\subsection{LH wave propagation effects}

To assess the effect of the magnetic configuration on the LH wave propagation, similar discharges with a density ramp were conducted in the USN and the Lower Single Null (LSN) configuration $\left(\mathrm{B}_{\mathrm{T}}=2.5 \mathrm{~T}, \mathrm{I}_{\mathrm{P}}=0.5 \mathrm{MA}\right)$ using 1.7 MW from the 4.6 GHz launcher. The electron cyclotron emission (ECE) from fast electrons driven by the LH wave, which is down-shifted by the relativistic effect and therefore measured at the very periphery of the plasma $(\mathrm{R}=2.30 \mathrm{~m}, \mathrm{r} / \mathrm{a}=1)$, was used as a qualitative measure of the difference in LHCD efficiency. The ECE signal was significantly larger in the USN configuration (around 30\% larger) in the whole density range explored, as seen in Figure 3. The fast electrons detected via ECE are likely to be in the energy range $50-100 \mathrm{keV}$, as this corresponds to an emission zone of $0.2<\mathrm{r} / \mathrm{a}<0.7$, which is compatible with C3PO/LUKE modelling of the LH wave deposition [3]. These fast electrons are also detected via the Hard X-ray diagnostic in EAST, showing that the bremsstrahlung in the hard X-ray range is higher in the USN configuration. The internal inductance is reduced in USN and the total energy content is found to be slightly higher $(+10 \%)$ for the USN configuration. In addition, the RF spectrum in the $4.58-4.61 \mathrm{GHz}$ range was recorded by a magnetic loop outside the vacuum vessel. Figure 4 shows that when the density exceeds $3 \times 10^{19} \mathrm{~m}^{-3}$, the width of the pump wave (at $\mathrm{f}=4.6 \mathrm{GHz}$ ) is larger for the LSN configuration. This could indicate that the wave number spectrum is affected, either by scattering on density fluctuations, or by a parametric decay into an ion-sound wave (f $\sim 2-3 \mathrm{MHz}$ ) [7].

In order to quantify the propagation effects and the resulting up-shift of the wave index $\left(\mathrm{n}_{/ /}\right)$on the LHCD efficiency, two discharges were performed in the same plasma configuration (identical to discharge \#64842) and the same power ( $0.4 \mathrm{MW}$ from the $4.6 \mathrm{GHz}$ launcher), launched by either the three lower rows of waveguides or by the three upper rows. The average reflection coefficient (RC) was very similar for the two discharges in the entire density range $(\mathrm{RC} \sim 3 \%)$, which indicates that the launched $n_{/ /}$spectra can be considered to be the same. It has to be noted that the directivity of the wave spectrum can have a considerable effect on the CD efficiency, as found in Tore Supra [12]. A slight difference on the loop voltage and the total stored energy at the benefit of the top launch was found. When the line averaged density $\left(\mathrm{n}_{\mathrm{e}}\right)$ exceeded $3.4 \times 10^{19} \mathrm{~m}^{-3}$, a strong interaction of the launcher with the edge plasma occured, which was also related to an increase of the gas injected by SMBI, and the stored energy decreased. 


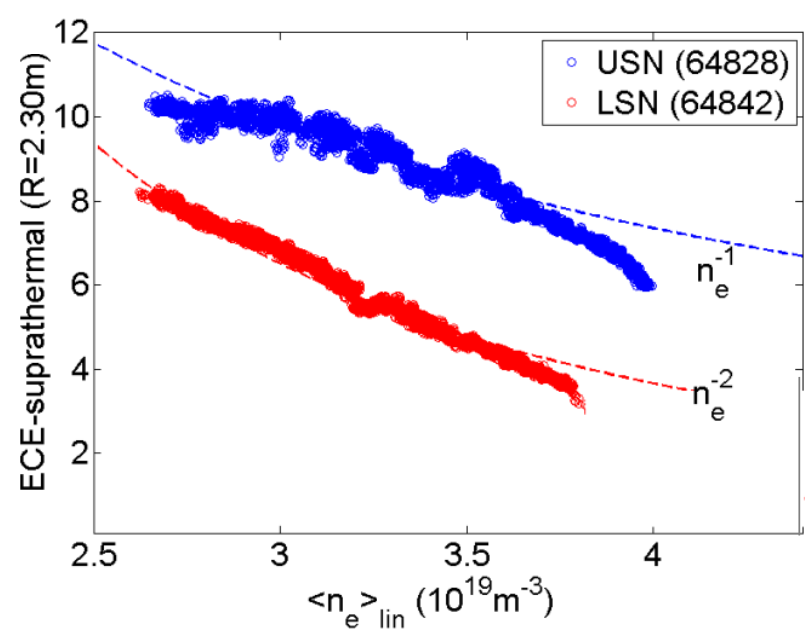

Fig. 3. Non-thermal Electron Cyclotron Emission for USN discharge \#64828 and LSN discharge \#64842 ( $\left.\mathrm{P}_{\mathrm{LH}}=1.7 \mathrm{MW}\right)$

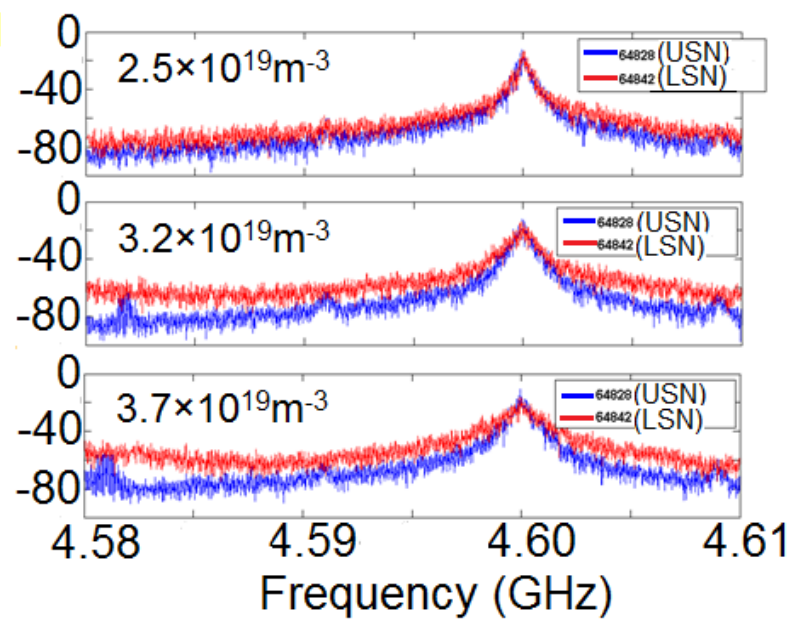

Fig. 4. RF spectra at three different densities for USN discharge \#64828 and LSN discharge \#64842 $\left(\mathrm{P}_{\mathrm{LH}}=1.7 \mathrm{MW}\right)$.

\subsection{Effect of LH launcher frequency}

In long pulse operation, both LHCD launchers are used in order to maximize the non-inductive current fraction and thus extend the pulse duration. H-mode discharges have been sustained by LHCD + ICRH $\left(\mathrm{P}_{\mathrm{LHCD}}=1.8-3.2\right.$ $\left.\mathrm{MW} ; \mathrm{P}_{\text {ICRH }}=1.2-1.5 \mathrm{MW}\right)$, in which most of the plasma current $\left(\mathrm{I}_{\mathrm{P}}=0.4 \mathrm{MA}\right)$ is non-inductively driven (loop voltage $<140 \mathrm{mV})$. At low loop voltage $\left(\mathrm{V}_{\mathrm{Loop}}\right)$, it can be shown that $\mathrm{V}_{\text {Loop }}$ varies almost linearly with $\mathrm{P}_{\text {norm, }}$ where $\mathrm{P}_{\text {norm }}=\mathrm{P}_{\mathrm{LHCD}} /\left(\mathrm{n}_{\mathrm{e}} \cdot \mathrm{R} \cdot \mathrm{I}_{\mathrm{P}}\right)$, and that the $\mathrm{CD}$ efficiency is given by $1 / \mathrm{P}_{\text {norm }}$ at $\mathrm{V}_{\text {Loop }}=0$ [13]. In the density range of interest, i.e. line averaged density $\mathrm{n}_{\mathrm{e}}=2.4-3.2 \times 10^{19}$ $\mathrm{m}^{-3}$, the loop voltage decreases linearly with $\mathrm{P}_{\text {norm }}$, only if the contribution of the power launched by the 2.45 $\mathrm{GHz}$ launcher to $\mathrm{P}_{\mathrm{LHCD}}$ is weighted by a factor 0.5 . Similar result was inferred from $\mathrm{V}_{\text {Loop }}$ measurements at modest LHCD power ( $\sim 1 \mathrm{MW})$, launched by the two launchers separately in the same discharge [7]. The same weighting also leads to less spread when fitting the hard $\mathrm{X}$-ray data and the total plasma energy. Neglecting the bootstrap current for these low beta discharges, the global efficiency is found to be close to $0.78 \times 10^{19}$ $\mathrm{A} /\left(\mathrm{W} \cdot \mathrm{m}^{2}\right)$.

The result above suggests that a fraction of the power launched from the $2.45 \mathrm{GHz}$ launcher may be damped at the plasma edge and is not entering the plasma core. This interpretation is partially consistent with RF spectrum measurements in the scrape-off layer, which indicate broader pump width for the $2.45 \mathrm{GHz}$ launcher [7]. Electron acceleration at the plasma edge due to Landau damping on high $\mathrm{n}_{/ /}$-components does not seem to be the cause, since infrared measurements of the divertor do not indicate higher heat load with the $2.45 \mathrm{GHz}$ launcher. In addition, calculations of the electron acceleration using the electric field from ALOHA [10] and a model for the electron dynamics in the LH electric field [14], show that the heat flux produced by edge electron acceleration is comparable for the two LH launchers [15].

\section{$4 \mathrm{H}$-mode experiments}

A study of the H-mode access with RF heating systems was carried out on EAST in 2016, in a plasma configuration as close as possible to that foreseen in WEST [4]. The maximum available heating power in these particular experiments was limited to $\sim 3.6 \mathrm{MW}$ $\left(\mathrm{P}_{\mathrm{LHCD}} \sim 2.8 \mathrm{MW}, \mathrm{P}_{\mathrm{ICRH}} \sim 0.4 \mathrm{MW}, \mathrm{P}_{\mathrm{ECRH}} \sim 0.4 \mathrm{MW}\right)$. The LHCD power (up to $2.8 \mathrm{MW}$ ) was mainly supplied by the $4.6 \mathrm{GHz}$ launcher (up to $2 \mathrm{MW}$ ), while the 2.45 $\mathrm{GHz}$ launcher gave up to $0.8 \mathrm{MW}$. H-mode plasmas lasting $6 \mathrm{~s}$, close to zero loop voltage $\left(\mathrm{V}_{\text {Loop }} \sim 50 \mathrm{mV}\right)$ could be achieved, as shown in Figure 5. The plasma configuration in the discharge in Figure 5 (\#64971) was LSN, i.e. with the strike point on the carbon divertor, and with $\mathrm{B} \times \nabla \mathrm{B}$ directed towards the target, which is expected to be favourable for the H-mode transition [16]. The $\mathrm{H}_{98(\mathrm{y} 2)}$-factor was found to be $\sim 0.76$ in \#64971. The $\mathrm{L}-\mathrm{H}$ transition occurred at $\mathrm{t}=2.2 \mathrm{~s}$, as characterized by the onset of Type I ELMs, after the application of 2.5 MW LHCD. The ECRH power was applied slightly later, at $\mathrm{t}=2.6 \mathrm{~s}$. In these experiments, it was found that low power ECRH and/or ICRH could be beneficial for sustaining the $\mathrm{H}$-mode. When changing the toroidal magnetic field direction so that $\mathrm{B} \times \nabla \mathrm{B}$ was directed away from the target, while keeping the plasma configuration and density the same, no H-mode transition was obtained even though the total injected power was 3.6 MW.

The same set of experiments was later repeated in the Upper Single Null (USN) configuration, i.e. with the strike points on the $\mathrm{W}$-divertor. One discharge with $\mathrm{B} \times \nabla \mathrm{B}$ directed upwards towards the $\mathrm{W}$-divertor, which again is expected to be favourable for the L-H transition, is shown in Figure 6. The H-mode is again sustained throughout the duration of the heating phase, at higher density and with similar $\mathrm{H}$-factor $\left(\mathrm{H}_{98(\mathrm{y} 2)} \sim 0.78\right)$ as in the shot in Figure 5. When reversing the $\mathrm{B} \times \nabla \mathrm{B}$ direction in USN configuration, and maintaining the same heating power as in \#65072 from the three systems (LHCD, ICRH and ECRH), only a short lasting H-mode ( $1 \mathrm{~s})$ was obtained, even though the heating power remained constant $(\sim 3.6 \mathrm{MW})$. 
In the discharges studied in this set of experiment $\left(\mathrm{I}_{\mathrm{P}}\right.$ $\left.=0.45 \mathrm{MA}, \mathrm{B}_{\mathrm{T}}= \pm 2.5 \mathrm{~T}\right)$, high density $\left(\mathrm{n}_{\mathrm{e}} \sim 3 \times 10^{19} \mathrm{~m}^{-3}\right)$ was required in most discharges to trigger the $\mathrm{H}$-mode. The additional power required to reach $\mathrm{H}$-mode was around 2.5 MW with $\mathrm{B} \times \nabla \mathrm{B}$ towards the target, and around 3.6 MW with $\mathrm{B} \times \nabla \mathrm{B}$ away from the target.

It could be noted that $\mathrm{H}$-modes were also obtained with LHCD alone at low power $\left(\mathrm{P}_{\mathrm{LHCD}} \sim 1.6 \mathrm{MW}\right)$, low density $\left(\mathrm{n}_{\mathrm{e}}=2.5 \times 10^{19} \mathrm{~m}^{-3}\right)$ and relatively high $\mathrm{H}$-factor $\left(\mathrm{H}_{98 \mathrm{y}} \sim 1.1\right)$, using the USN configuration and $\mathrm{B} \times \nabla \mathrm{B}$ directed upwards. However, these discharges transited back to L-mode after approximately $2 \mathrm{~s}$.

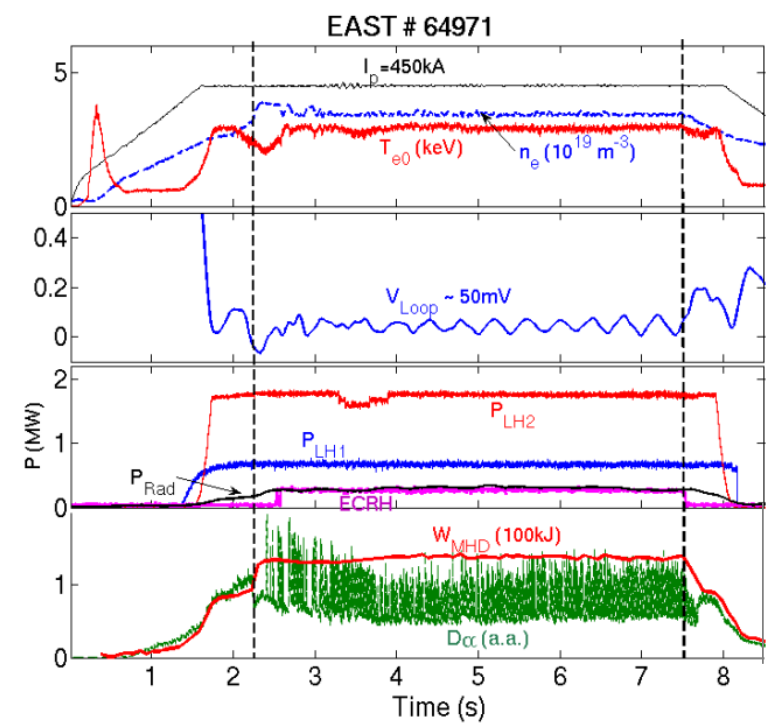

Fig. 5. Time traces of H-mode discharge in LSN configuration, $\mathrm{B}_{\mathrm{T}}=-2.5 \mathrm{~T}$ and $\mathrm{B} \times \nabla \mathrm{B}$ directed downwards, towards the target .

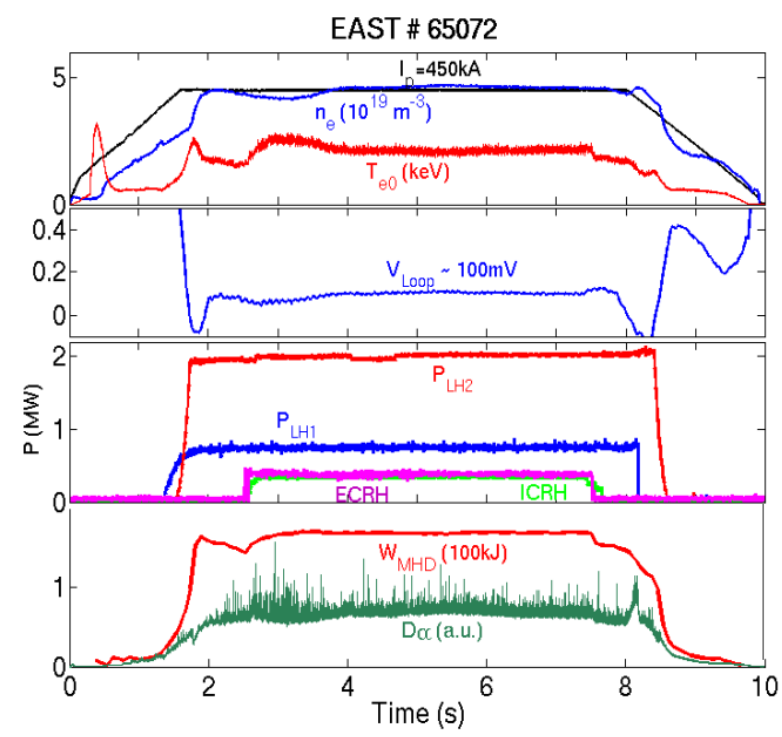

Fig. 6. H-mode discharge in USN configuration (W-divertor), $\mathrm{B}_{\mathrm{T}}=2.5 \mathrm{~T}$ and $\mathrm{B} \times \nabla \mathrm{B}$ directed upwards, towards the target.

\section{Summary}

Several experiments have been carried out on EAST with the goal to optimizing the efficiency of the LHCD systems in view of long pulse H-mode operation. The
LHCD experiments indicate that the USN configuration could be more favourable than the LSN for the current drive efficiency. The different gas feeding location tested (high field side, low field side and upper divertor) do not affect the LH wave coupling differently. However, gas fueling with SMBI has shown to reduce the reflection coefficient, due the increase of density in the scrape-off layer, linked to increased radial transport during SMBI.

During H-mode experiments, $6 \mathrm{~s}$ long H-modes were obtained with loop voltage as low as $50 \mathrm{mV}$, using LHCD as main heating and current drive source, accompanied by low power ECRH and/or ICRH. Easier access to $\mathrm{H}$-mode was indeed found when the $\mathrm{B} \times \nabla \mathrm{B}$ drift was directed towards the target. This was confirmed in both LSN (carbon divertor) and USN (W-divertor) configurations. In summary, all results obtained here also give valuable information in view of the exploitation of the WEST tokamak, which has similar size to EAST, and which will use RF heating methods only in $\mathrm{W}$-environment.

The CEA/IRFM members warmly acknowledge the hospitality of the ASIPP Team during the visits to EAST. This work is supported by the National Magnetic Confinement Fusion Program of China (Grant No 2015GB10200) and the Associated Laboratory CEA/IRFM - CAS/ASIPP. This work has been carried out within the framework of the EUROfusion Consortium and has received funding from the European research and training programme under grant agreement $\mathrm{N}^{\circ}$ 633053. The views and opinions expressed herein do not necessarily reflect those of the European Commission.

\section{References}

1. B. Wan et al., Nucl. Fusion 57, 102019 (2017)

2. F.K. Liu et al., this conference

3. Y. Peysson et al., this conference

4. C. Bourdelle et al., Nucl. Fusion 55, 063017 (2015)

5. E.H. Kong et al., Plasma Phys. Control. Fusion 56, 075016 (2014)

6. F.K. Liu et al., Nucl. Fusion 55, 123022 (2015)

7. M.H. Li et al., Phys. Plasmas 23, 102512 (2016)

8. Y. Peysson and J. Decker, Fusion Sci. Technol. 65, $22(2014)$

9. Y.L. Li et al., this conference

10. J. Hillairet et al., Nucl. Fusion 50, 125010 (2010)

11. X.L. Zou et al., Proc. 24th IAEA FEC (2012), San Diego, CA, paper PD/P8-08

12. E. Nilsson et al., Nucl. Fusion 53, 083018 (2013)

13. M. Goniche et al., Phys. Plasmas 21, 061515 (2014)

14. V. Fuchs et al., Nucl. Fusion 43, 341 (2003)

15. L. Valade et al., AIP Conf. Proc. 1689, 080007 (2015)

16. G.R. McKee et al., Nucl. Fusion 49, 115016 (2009) 